\title{
Factors Affecting Health-Related Quality of Life in Multimorbidity
}

\author{
Eunmi Lee ${ }^{1} \mathbb{C}$, Sunkyung Cha ${ }^{2, *(\mathbb{D})}$ and Geun Myun Kim ${ }^{3}$ \\ 1 Department of Nursing, Hoseo University, Asan 31499, Korea; sweetbear2@hanmail.net \\ 2 Department of Nursing Science, Sun Moon University, Asan 31460, Korea \\ 3 Department of Nursing, Gangneung-Wonju National University, Wonju 26403, Korea; gmkim@gwnu.ac.kr \\ * Correspondence: skc0701@hanmail.net; Tel.: +82-41-530-2743; Fax: +82-41-530-2725
}

check for

updates

Citation: Lee, E.; Cha, S.; Kim, G.M. Factors Affecting Health-Related Quality of Life in Multimorbidity. Healthcare 2021, 9, 334. https:// doi.org/10.3390/healthcare9030334

Academic Editor: Phyo Kyaw Myint

Received: 24 February 2021

Accepted: 12 March 2021

Published: 16 March 2021

Publisher's Note: MDPI stays neutral with regard to jurisdictional claims in published maps and institutional affiliations.

Copyright: (c) 2021 by the authors. Licensee MDPI, Basel, Switzerland. This article is an open access article distributed under the terms and conditions of the Creative Commons Attribution (CC BY) license (https:// creativecommons.org/licenses/by/ $4.0 /)$.

\begin{abstract}
We investigated the effect of predisposing, enabling, need factors, and health behaviors on health-related quality of life (HRQoL) of patients with multimorbidity according to Andersen's model. This study is a secondary analysis of population-based cross-sectional surveys. Data from 328 patients with multimorbidity ( $\geq 3$ co-occurring chronic diseases) from the 6 th $/ 7$ th Korea National Health and Nutrition Examination Surveys were analyzed using logistic regression. Patients $\geq 65$ years, without private insurance, with poor subjective health, unmet medical needs, and/or limited activity were more likely to experience mobility problems. Self-care problems were more likely among those without private insurance and/or with limited activity. Patients lacking living security, with poor subjective health, limited activity, and/or who smoked were more likely to experience problems performing usual activities. Pain/discomfort was more likely among females, Medicaid beneficiaries, and patients with limited activity and/or with poor subjective health. Patients with poor subjective health, limited activity, and/or unmet medical needs were more likely to experience anxiety/depression. The investigation of HRQoL in multimorbidity should consider predisposing, enabling, need factors, and health behaviors. Interventions addressing movement restrictions and personalized care based on HRQoL domains should be prioritized.
\end{abstract}

Keywords: multimorbidity; Andersen's model; health-related quality of life; chronic disease management

\section{Introduction}

\subsection{Background}

As medical technology advances and human life expectancy increases, the prevalence of chronic diseases has raised as a result of population aging. Chronic diseases are a leading cause of disability and death worldwide, accounting for $60 \%$ of global deaths [1]. However, the management of health-related habits such as smoking, drinking alcohol, physical activity, and diet, which contribute to chronic diseases, has not improved. The prevalence of multimorbidity is also on the rise, and the OECD recently identified chronic disease as a critical issue faced by many countries [2]. Multimorbidity refers to two or more co-occurring diseases [3-5] and is prevalent in $23 \%$ of the general population, affecting $65 \%$ of individuals aged $\geq 65$ years, and $55 \%$ of patients with chronic diseases [6].

In South Korea, the average patient with chronic disease aged $\geq 50$ years has two or more diseases. On average, patients aged $\geq 60$ years have 3 , and patients aged $\geq 70$ years have 3.5 conditions [7]. Multimorbidity is expected to increase with population aging [8], thus requiring the implementation of health-related policies and continuous management of co-occurring diseases, with attendant rising treatment frequency and medical costs [9]. In South Korea, the focus of multimorbidity studies has been on their prevalence, and a few studies have focused on chronic disease combinations and the cost of medical treatments related to multimorbidity $[8,10]$. 
Patients with multimorbidity must receive continuous treatment and maintain their quality of life (QoL). The QoL of older adults is especially important when considering the prevalence of multimorbidity in isolation; however, research using samples of younger adults is also needed, since there may be a higher prevalence of multimorbidity among adults aged $<65$ years depending on the population distribution ratio in a given population group $[8,11]$. Therefore, there is a need to examine QoL in all adults with multimorbidity.

Health-related QoL (HRQoL) is a subjective, multidimensional concept expressing an individual's satisfaction with functional abilities related to his/her health [12]. It is useful for determining individuals' functional abilities and wellness [13], and as an index for policies in the public health sector when determining and improving community residents ${ }^{\prime}$ health status. Most research on the factors influencing HRQoL has focused on individual characteristics, such as financial status, education level, occupation, emotional state (e.g., depression, anxiety, stress), and physical health, in vulnerable elderly populations such as those living alone or receiving a basic income [14-17]. Thus, applying the results of these studies to patients with multimorbidity has limitations. Although research has reported that HRQoL is affected by the interaction of individuals' various characteristics, including emotional state and socio-environmental factors, research comprehensively examining the physical, mental, and social aspects of QoL in patients with multimorbidity has not been conducted in South Korea.

Andersen's model was developed in the 1990s to analyze and explain health outcomes such as customer satisfaction or QoL in relation to health services [18], and suggests that predisposing, enabling, and needs factors influence individuals' health outcomes. In Korea, Andersen's model has usually been used in QoL research with older adults [19-21]. Additionally, the model was designed to explore how and why individuals and families utilize general healthcare, welfare services in socially structured environments, and the effect on outcomes, including health status and health satisfaction. The model is multidimensional and includes contextual and individual characteristics as well as health behaviors and outcomes [22].

According to Andersen's model, the primary factors determining health behaviors are socio-demographic characteristics, public health systems, and external environmental factors, which also affect health outcomes such as consumer satisfaction and QoL [23,24]. Studies conducted in Korea based on this model include those examining older adults' ability to perform usual daily activities and use medical services, and their oral HRQoL [21,24,25]. In a systematic review about health service use by Babitsch et al. (2012), age, marital status, gender, education level, and race were identified as predisposing factors; household income, financial condition, health insurance availability, and usable resources were enabling factors; and health condition, subjective health status, and other various diseases were need factors. This study aimed to identify factors influencing each dimension of HRQoL in patients with multimorbidity in Korea using Andersen's model. In the future, this information could be used as a basis for establishing health and welfare policies related to multimorbidity.

\subsection{Purpose}

In this study, we investigated the effect individual characteristics as primary factors (i.e., predisposing, enabling, and need factors) and health activities as secondary factors on HRQoL in patients with multimorbidity based on the application of Andersen's model.

\section{Methods}

2.1. Design

A cross-sectional survey was used to identify factors affecting the HRQoL of patients with multimorbidity (i.e., three or more co-occurring chronic diseases) based on Andersen's model. 


\subsection{Participants}

We analyzed data collected in 2014 and 2015 in the 6th Korea National Health and Nutrition Examination Survey (KNHNES) (2013-2015) and in 2016 in the 7th KNHNES (2016-2018). These surveys assess health status, health behavior, food consumption, and nutritional status of all Korean nationals over the age of 1 year. The KNHNES is a singleyear survey conducted over 3 years. It was initiated in 1998 and has been used as an annual rolling sample survey method since 2007 to help establish public health policies, involving setting goals for and evaluating the comprehensive national health improvement plan, and developing other health improvement plans [26]. In this study, data from the most recent 3 years $(2014,2015$, and 2016) were used.

Among the 27 chronic diseases studied in the KNHNES, respondents with at least 3 of the 13 chronic diseases defined by the World Health Organization (WHO) (i.e., hypertension, stroke, cardiac infarction, angina, diabetes, and stomach, liver, colon, breast, cervical, lung, thyroid, and other cancers) occurring concurrently were defined as having multimorbidity. In 2014, the second year of the 6th survey (2013-2015), 7550 responses were recorded; 7380 responses were recorded in 2015, the third year of the 6th survey; and 8150 responses were recorded in 2016, the first year of the 7th survey (2016-2018). The number of patients with at least three chronic diseases [5] was 93, 119, and 116 in 2014, 2015, and 2016, respectively. The total number of respondents whose data were used in this study was thus 328 (Figure 1).

\begin{tabular}{|c|c|c|c|}
\hline Survey series & Survey year & Responses & Multimorbidity \\
\hline $6^{\text {th }}(2013-2015)$ & 2014 & 7550 & 93 \\
\hline $6^{\text {th }}(2013-2015)$ & 2015 & 7380 & 119 \\
\hline $7^{\text {th }}(2016-2018)$ & 2016 & 8150 & 116 \\
\hline total & & 23030 & 328 \\
\hline
\end{tabular}

Figure 1. Respondents of patient with multimorbidity $(N=328)$ (South Korea, 2014-2016).

\subsection{Measurements}

HRQoL (dependent variable), was examined using the EQ-5D-3L after the KCDC obtained permission from the EuroQol Group, and comprised five dimensions: mobility, self-care, usual activities, pain/discomfort, and anxiety/depression (emotional state: e.g., depression, anxiety, stress). Items are rated on a 3-point scale $(1=$ "no problem", 2 = "some problem", and $3=$ "extreme problem"). In this study, each HRQoL domain was assessed as a dichotomous variable: "no problem" and "problem".

In the Andersen model, contextual characteristics and individual characteristics are factors influencing the use of medical services along with health conditions, which are defined as predisposing factors, enabling factors, and need factors. Predisposing factors are inherent socio-demographic characteristics, such as gender, age, education level, and marital status. Enabling factors are those facilitating or inhibiting the use of medical service or health conditions, include the receipt of basic living security, health insurance type, existence of private insurance, household income, living with family, and limited activity. Need factors are elements that require medical services such as the presence of a disease and include the number of chronic diseases, subjective health, and the presence of unmet medical needs. Lastly, health behaviors are health-related activities, such as smoking, drinking alcohol, physical activity, and health screenings.

The model factors were categorized into predisposing, enabling, and need factors, and health behaviors. Gender, age $(<65, \geq 65)$, education level ( $\leq$ high school graduate, $>$ high school graduate), and marital status (presence or absence of spouse) were considered as predisposing factors. Receipt of basic living security, insurance type (national health insurance, Medicaid), private insurance household income (in 10,000 South Korean won), 
living with family, and limited activity were considered enabling factors. The number of chronic diseases, subjective health status (good or poor), and having unmet medical needs were considered as need factors. Lastly, smoking, drinking, physical activity, and health screenings were considered as health behaviors.

\subsection{Statistical Analysis}

SPSS 20.0 was used to analyze the data. Frequency analysis and descriptive statistics were performed for all variables as general characteristics. Logistic regression analysis was conducted to identify the factors affecting each HRQoL dimension (dependent variables); Andersen's model variables were added in the following order: predisposing factors, enabling factors, need factors, and health behaviors.

\section{Results}

\subsection{General Characteristics of Multimorbidity}

Participants' general characteristics are shown in Table 1. Regarding predisposing factors, 164 (50\%) respondents were male; $240(73.2 \%)$ were $\geq 65$ years $(26.8 \%) ; 284(87.6 \%)$ were $\leq$ high school graduate, while $40(12.45 \%)$ were $>$ high school graduate; $225(68.6 \%)$ had a spouse.

Regarding enabling factors, $277(84.5 \%)$ respondents had never received basic living security. Most respondents $(281,87.85 \%)$ had national health insurance, and $39(12.2 \%)$ had Medicaid; moreover, 117 (35.7\%) had private insurance, and 211 (64.3\%) did not have any. The average household income was 2,101,000 won per month; 257 (78.3\%) respondents were living with family; $89(27.1 \%)$ respondents displayed limited activity.

Regarding need factors, 275 (83.9\%) respondents had three chronic diseases, while $46(14.0 \%)$ had four, and $7(2.15 \%)$ had five (mean number of diseases $=3.2) ; 205(62.5 \%)$ respondents had poor subjective health status; and 41 (12.5\%) respondents had unmet medical needs.

Regarding health behaviors, 59 (18.4\%) respondents smoked; 153 (47.7\%) drank alcohol; $42(12.9 \%)$ respondents exercised; and 191 (58.4\%) underwent health screenings.

Regarding the HRQoL domains, 174 (53.2\%) respondents had mobility problems, 72 $(22.0 \%)$ had self-care problems, $141(43.0 \%)$ had problems with usual activities, $152(46.3 \%)$ had pain/discomfort, and 88 (26.8\%) had anxiety/depression. 
Table 1. General characteristics of patients with multimorbidity $(N=328)$ (South Korea, 2014-2016).

\begin{tabular}{|c|c|c|c|c|}
\hline & able & Class & $n(\%)$ & Mean \pm SD \\
\hline \multirow{9}{*}{ Predisposing factors } & \multirow[b]{2}{*}{ Gender } & Male & $164(50.0)$ & \\
\hline & & Female & $164(50.0)$ & \\
\hline & \multirow{2}{*}{ Age } & Under 65 & $88(26.8)$ & \\
\hline & & 65 years or older & $240(73.2)$ & \\
\hline & \multirow{3}{*}{ Education level } & Below high school & $284(87.6)$ & \\
\hline & & graduate & & \\
\hline & & $\begin{array}{l}\text { Above high school } \\
\text { graduate }\end{array}$ & $40(12.4)$ & \\
\hline & \multirow[b]{2}{*}{ Marital status } & With spouse & $225(68.6)$ & \\
\hline & & No spouse & $103(31.4)$ & \\
\hline \multirow{15}{*}{ Enabling factors } & \multirow{2}{*}{ Basic living security } & No & $277(84.5)$ & \\
\hline & & Yes & $51(15.5)$ & \\
\hline & \multirow{3}{*}{ Health insurance } & National Health & $281(87.8)$ & \\
\hline & & Insurance & $201(07.0)$ & \\
\hline & & Medicaid & $39(12.2)$ & \\
\hline & \multirow{3}{*}{ Private insurance } & Yes & $117(35.7)$ & \\
\hline & & No & $211(64.3)$ & \\
\hline & & Low & $161(49.1)$ & \\
\hline & \multirow{3}{*}{ Household income } & Mid-low & $81(24.7)$ & \\
\hline & & Mid-high & $50(15.2)$ & $210.1 \pm 244.8^{\mathrm{a}}$ \\
\hline & & High & $36(11.0)$ & \\
\hline & \multirow{2}{*}{ Living with family } & Yes & $257(78.3)$ & \\
\hline & & No & $71(21.7)$ & \\
\hline & \multirow{2}{*}{ Limited activity } & No & $239(72.9)$ & \\
\hline & & Yes & $89(27.1)$ & \\
\hline \multirow{7}{*}{ Need factors } & \multirow{3}{*}{$\begin{array}{c}\text { Number of chronic } \\
\text { diseases }\end{array}$} & 3 & $275(83.9)$ & \\
\hline & & 4 & $46(14.0)$ & $3.2 \pm 0.4$ \\
\hline & & 5 & $7(2.1)$ & \\
\hline & \multirow{2}{*}{ Subjective health status } & Good & $123(37.5)$ & \\
\hline & & Poor & $205(62.5)$ & \\
\hline & \multirow{2}{*}{ Unmet medical needs } & No & $287(87.5)$ & \\
\hline & & Yes & $41(12.5)$ & \\
\hline \multirow{8}{*}{ Health behaviors } & \multirow{2}{*}{ Smoking } & No & $262(81.6)$ & \\
\hline & & Yes & $59(18.4)$ & \\
\hline & \multirow{2}{*}{ Drinking } & No & $168(52.3)$ & \\
\hline & & Yes & $153(47.7)$ & \\
\hline & \multirow{2}{*}{ Physical activity } & Yes & $42(12.9)$ & \\
\hline & & No & $283(87.1)$ & \\
\hline & \multirow{2}{*}{ Health screenings } & Yes & $191(58.4)$ & \\
\hline & & No & $136(41.6)$ & \\
\hline \multirow{10}{*}{ EQ-5D-L3 } & & No problem & $153(46.8)$ & \\
\hline & Mobility & Problem & $174(53.2)$ & \\
\hline & & No problem & $256(78.0)$ & \\
\hline & Self-care & Problem & $72(22.0)$ & \\
\hline & & No problem & $187(57.0)$ & \\
\hline & Usual activity & Problem & $141(43.0)$ & \\
\hline & & No problem & $176(53.7)$ & \\
\hline & Pain/discomfort & Problem & $152(46.3)$ & \\
\hline & & No problem & $240(73.2)$ & \\
\hline & Anxiety/depression & Problem & $88(26.8)$ & \\
\hline
\end{tabular}

a 10,000 South Korean won; SD: standard deviation. 


\subsection{Factors Affecting $H R Q o L$}

In terms of factors affecting the mobility dimension of HRQoL (Table 2), respondents $\geq 65$ years, without high school education, and/or without a spouse were more likely to have problems with mobility in Model 1 (predisposing factors). Respondents aged $\geq 65$ years, without high school education, without private insurance, and/or with limited activity were more likely to have problems with mobility in Model 2 (predisposing + enabling factors). Respondents aged $\geq 65$ years, without high school education, without private insurance, with limited activity, who had poor subjective health status, and/or who had unmet medical needs were more likely to have problems with mobility in Model 3 (predisposing + enabling + need factors). Respondents $\geq 65$ years, without private insurance, with limited activity, who had poor subjective health status, and/or who had unmet medical needs were more likely to have problems with mobility in Model 4 (predisposing factors + enabling factors + need factors + health behaviors).

In terms of factors affecting the self-care dimension of HRQoL (Table 3), Model 2-4, respondents without private insurance and/or who had limited activity were more likely to have problems with self-care, while other factors were not significant.

Regarding factors affecting the usual activities dimension of HRQoL (Table 4), Respondents who received Medicaid, without private insurance, and/or with limited activity were more likely to have problems with usual activities in Model 2. In Model 3, respondents who had never received basic living security, who had Medicaid, who had limited activity, and/or who had poor subjective health status were more likely to have problems with usual activities. In Model 4, respondents who had never received basic living security, with limited activity, who had poor subjective health status, and/or who smoked were more likely to have problems with usual activities.

In terms of factors affecting pain/discomfort dimension of HRQoL (Table 5), females were more likely to experience problems than males in Model 1. Respondents who were female, who received Medicaid, and/or who had limited activity were more likely to experience pain/discomfort in Model 2. In Models 3 and 4, respondents who were female, who received Medicaid, who had limited activity, and/or who had poor subjective health status were more likely to experience pain/discomfort.

In terms of factors affecting the anxiety/depression dimension of HRQoL (Table 6), respondents who were $<65$ years and/or had limited activity were more likely to experience anxiety/depression in Model 2. In both Models 3 and 4, respondents with limited activity, who had poor subjective health status, and/or who had unmet medical needs were more likely to experience anxiety/depression. 
Table 2. Factors affecting mobility dimension of health-related quality of life in patients with multimorbidity according to Andersen's model (South Korea, 2014-2016).

\begin{tabular}{|c|c|c|c|c|c|}
\hline \multirow{2}{*}{\multicolumn{2}{|c|}{ Variable }} & Model 1 & Model 2 & Model 3 & Model 4 \\
\hline & & $\begin{array}{c}\text { OR }(p) \\
(95 \% \mathrm{CI})\end{array}$ & $\begin{array}{c}\text { OR }(p) \\
(95 \% \text { CI })\end{array}$ & $\begin{array}{c}\text { OR }(p) \\
(95 \% \text { CI })\end{array}$ & $\begin{array}{c}\text { OR }(p) \\
(95 \% \text { CI })\end{array}$ \\
\hline \multirow{4}{*}{$\begin{array}{l}\text { Predisposing } \\
\text { factors }\end{array}$} & Gender (ref = Male) & $\begin{array}{l}1.06(0.820) \\
(0.65,1.72)\end{array}$ & $\begin{array}{l}1.12(0.661) \\
(0.67,1.88)\end{array}$ & $\begin{array}{l}1.04(0.888) \\
(0.61,1.77)\end{array}$ & $\begin{array}{l}1.21(0.543) \\
(0.66,2.19)\end{array}$ \\
\hline & Age (ref = Under 65) & $\begin{array}{l}2.48(0.001) \\
(1.47,4.18)\end{array}$ & $\begin{array}{l}1.95(0.026) \\
(1.08,3.51)\end{array}$ & $\begin{array}{l}2.33(0.007) \\
(1.26,4.31)\end{array}$ & $\begin{array}{l}2.30(0.011) \\
(1.21,4.37)\end{array}$ \\
\hline & $\begin{array}{c}\text { Education level } \\
\text { (ref = Below high school } \\
\text { graduate) }\end{array}$ & $\begin{array}{l}0.28(0.001) \\
(0.13,0.61)\end{array}$ & $\begin{array}{l}0.31(0.006) \\
(0.14,0.72)\end{array}$ & $\begin{array}{l}0.36(0.019) \\
(0.15,0.84)\end{array}$ & $\begin{array}{l}0.43(0.067) \\
(0.18,1.06)\end{array}$ \\
\hline & Marital status (ref $=$ With spouse) & $\begin{array}{l}1.80(0.028) \\
(1.07,3.04)\end{array}$ & $\begin{array}{l}1.28(0.503) \\
(0.62,2.62)\end{array}$ & $\begin{array}{l}1.37(0.407) \\
(0.65,2.88)\end{array}$ & $\begin{array}{l}1.22(0.621) \\
(0.56,2.66)\end{array}$ \\
\hline \multirow{6}{*}{ Enabling factors } & Basic living security (ref = No) & & $\begin{array}{l}0.87(0.775) \\
(0.32,2.33)\end{array}$ & $\begin{array}{l}0.77(0.629) \\
(0.27,2.21)\end{array}$ & $\begin{array}{l}0.77(0.627) \\
(0.27,2.22)\end{array}$ \\
\hline & $\begin{array}{l}\text { Health insurance }(\text { ref }=\text { National } \\
\text { health insurance) }\end{array}$ & & $\begin{array}{l}2.32(0.142) \\
(0.75,7.17)\end{array}$ & $\begin{array}{l}2.48(0.135) \\
(0.75,8.17)\end{array}$ & $\begin{array}{l}2.31(0.171) \\
(0.70,7.68)\end{array}$ \\
\hline & Private insurance (ref = Yes) & & $\begin{array}{l}1.85(0.029) \\
(1.07,3.20)\end{array}$ & $\begin{array}{l}1.83(0.035) \\
(1.04,3.20)\end{array}$ & $\begin{array}{l}1.85(0.037) \\
(1.04,3.30)\end{array}$ \\
\hline & $\begin{array}{l}\text { Household income (monthly } \\
\text { average) }\end{array}$ & & $\begin{array}{l}1.00(0.950) \\
(1.00,1.00)\end{array}$ & $\begin{array}{l}1.00(0.804) \\
(1.00 .1 .00)\end{array}$ & $\begin{array}{l}1.00(0.594) \\
(1.00,1.00)\end{array}$ \\
\hline & Living with family (ref = Yes) & & $\begin{array}{l}1.37(0.455) \\
(0.60,3.12)\end{array}$ & $\begin{array}{l}1.44(0.397) \\
(0.62,3.36)\end{array}$ & $\begin{array}{l}1.59(0.296) \\
(0.67,3.81)\end{array}$ \\
\hline & Limited activity $(\mathrm{ref}=\mathrm{No})$ & & $\begin{array}{l}3.26(<0.001) \\
(1.82,5.86)\end{array}$ & $\begin{array}{l}2.31(0.008) \\
(1.24,4.31)\end{array}$ & $\begin{array}{l}2.43(0.007) \\
(1.27,4.62)\end{array}$ \\
\hline \multirow{3}{*}{ Need factors } & Number of chronic diseases & & & $\begin{array}{l}1.19(0.569) \\
(0.66,2.12)\end{array}$ & $\begin{array}{l}1.20(0.552) \\
(0.66,2.19)\end{array}$ \\
\hline & $\begin{array}{l}\text { Subjective health status }(\text { ref }= \\
\text { Good) }\end{array}$ & & & $\begin{array}{l}2.00(0.012) \\
(1.17,3.42)\end{array}$ & $\begin{array}{l}1.78(0.042) \\
(1.02,3.10)\end{array}$ \\
\hline & Unmet medical needs $(\mathrm{ref}=\mathrm{No})$ & & & $\begin{array}{l}2.47(0.044) \\
(1.03,5.93)\end{array}$ & $\begin{array}{l}2.78(0.025) \\
(1.14,6.79) \\
\end{array}$ \\
\hline \multirow{4}{*}{ Health behaviors } & Smoking $(\mathrm{ref}=\mathrm{No})$ & & & & $\begin{array}{l}1.95(0.075) \\
(0.94,4.08)\end{array}$ \\
\hline & Drinking $(\mathrm{ref}=\mathrm{No})$ & & & & $\begin{array}{l}0.80(0.437) \\
(0.46,1.41)\end{array}$ \\
\hline & Physical activity (ref = Yes) & & & & $\begin{array}{l}2.19(0.073) \\
(0.93,5.16)\end{array}$ \\
\hline & Health screenings $(\mathrm{ref}=\mathrm{Yes})$ & & & & $\begin{array}{l}1.36(0.260) \\
(0.80,2.33)\end{array}$ \\
\hline & Wald $(p)$ & $28.41(<0.001)$ & $47.31(<0.001)$ & $54.42(<0.001)$ & $57.51(<0.001)$ \\
\hline & Nagelkerke's $\mathrm{R}^{2}$ & 0.130 & 0.237 & 0.281 & 0.308 \\
\hline
\end{tabular}


Table 3. Factors affecting self-care dimension of health-related quality of life in patients with multimorbidity according to Andersen's model (South Korea, 2014-2016).

\begin{tabular}{|c|c|c|c|c|c|}
\hline \multirow{2}{*}{\multicolumn{2}{|c|}{ Variable }} & Model 1 & Model 2 & Model 3 & Model 4 \\
\hline & & $\begin{array}{l}\text { OR }(p) \\
(95 \% \mathrm{CI})\end{array}$ & $\begin{array}{c}\text { OR }(p) \\
(95 \% \text { CI })\end{array}$ & $\begin{array}{c}\text { OR }(p) \\
(95 \% \text { CI })\end{array}$ & $\begin{array}{c}\text { OR }(p) \\
(95 \% \text { CI })\end{array}$ \\
\hline \multirow{4}{*}{$\begin{array}{l}\text { Predisposing } \\
\text { factors }\end{array}$} & Gender (ref = Male) & $\begin{array}{l}0.78(0.397) \\
(0.44,1.38)\end{array}$ & $\begin{array}{l}0.88(0.675) \\
(0.47,1.63)\end{array}$ & $\begin{array}{l}0.83(0.557) \\
(0.44,1.55)\end{array}$ & $\begin{array}{l}0.99(0.970) \\
(0.48,2.05)\end{array}$ \\
\hline & Age (ref = Under 65) & $\begin{array}{l}1.82(0.079) \\
(0.93,3.53)\end{array}$ & $\begin{array}{l}1.07(0.862) \\
(0.50,2.30)\end{array}$ & $\begin{array}{l}1.15(0.722) \\
(0.53,2.54)\end{array}$ & $\begin{array}{l}1.26(0.583) \\
(0.55,2.85)\end{array}$ \\
\hline & $\begin{array}{c}\text { Education level } \\
(\text { ref = Below high school } \\
\text { graduate })\end{array}$ & $\begin{array}{l}0.50(0.170) \\
(0.19,1.35)\end{array}$ & $\begin{array}{l}0.77(0.629) \\
(0.26,2.26)\end{array}$ & $\begin{array}{l}0.78(0.668) \\
(0.25,2.41)\end{array}$ & $\begin{array}{l}0.70(0.559) \\
(0.22,2.29)\end{array}$ \\
\hline & Marital status (ref = With spouse) & $\begin{array}{l}1.57(0.134) \\
(0.87,2.82)\end{array}$ & $\begin{array}{l}1.50(0.375) \\
(0.61,3.64)\end{array}$ & $\begin{array}{l}1.48(0.392) \\
(0.61,3.61)\end{array}$ & $\begin{array}{l}1.16(0.757) \\
(0.45,3.02)\end{array}$ \\
\hline \multirow{7}{*}{ Enabling factors } & Basic living security (ref = No) & & $\begin{array}{l}0.56(0.312) \\
(0.18,1.72)\end{array}$ & $\begin{array}{l}0.54(0.303) \\
(0.17,1.75)\end{array}$ & $\begin{array}{l}0.52(0.282) \\
(0.15,1.73)\end{array}$ \\
\hline & $\begin{array}{l}\text { Health insurance (ref = National } \\
\text { health insurance) }\end{array}$ & & $\begin{array}{l}1.25(0.708) \\
(0.39,3.97)\end{array}$ & $\begin{array}{l}1.10(0.875) \\
(0.33,3.71)\end{array}$ & $\begin{array}{l}0.89(0.849) \\
(0.25,3.14)\end{array}$ \\
\hline & Private insurance $($ ref $=$ Yes $)$ & & $\begin{array}{l}2.58(0.014) \\
(1.12,5.49)\end{array}$ & $\begin{array}{l}2.69(0.011) \\
(1.25,5.80)\end{array}$ & $\begin{array}{l}2.76(0.010) \\
(1.27,6.01)\end{array}$ \\
\hline & \multirow{2}{*}{$\begin{array}{l}\text { Household income (monthly } \\
\text { average) }\end{array}$} & & $1.00(0.099)$ & $1.00(0.172)$ & $1.00(0.234)$ \\
\hline & & & $(1.00,1.00)$ & $(1.00,1.00)$ & $(1.00,1.00)$ \\
\hline & Living with family (ref = Yes) & & $\begin{array}{l}0.82(0.700) \\
(0.31,2.20)\end{array}$ & $\begin{array}{l}0.93(0.889) \\
(0.35,2.50)\end{array}$ & $\begin{array}{l}1.20(0.725) \\
(0.43,3.32)\end{array}$ \\
\hline & Limited activity (ref = No) & & $\begin{array}{l}4.21(<0.001) \\
(2.33,7.60)\end{array}$ & $\begin{array}{c}3.59(<0.001) \\
(1.91,6.73)\end{array}$ & $\begin{array}{l}4.15(<0.001) \\
(2.15,8.01)\end{array}$ \\
\hline \multirow{3}{*}{ Need factors } & Number of chronic diseases & & & \multirow{3}{*}{$\begin{array}{l}0.65(0.229) \\
(0.32,1.32) \\
1.96(0.059) \\
(0.98,3.92) \\
1.92(0.130) \\
(0.83,4.46)\end{array}$} & $\begin{array}{l}0.65(0.234) \\
(0.32,1.33)\end{array}$ \\
\hline & $\begin{array}{l}\text { Subjective health status }(\mathrm{ref}= \\
\text { Good) }\end{array}$ & & & & $\begin{array}{l}1.78(0.112) \\
(0.87,3.64)\end{array}$ \\
\hline & Unmet medical needs $(\mathrm{ref}=\mathrm{No})$ & & & & $\begin{array}{l}2.12(0.094) \\
(0.88,5.08)\end{array}$ \\
\hline \multirow{4}{*}{ Health behaviors } & Smoking $(\mathrm{ref}=\mathrm{No})$ & & & & $\begin{array}{l}1.73(0.201) \\
(0.75,4.02)\end{array}$ \\
\hline & Drinking $(\mathrm{ref}=\mathrm{No})$ & & & & $\begin{array}{l}0.94(0.869) \\
(0.46,1.92)\end{array}$ \\
\hline & Physical activity (ref = Yes) & & & & $\begin{array}{l}0.69(0.449) \\
(0.26,1.82)\end{array}$ \\
\hline & Health screenings $($ ref $=$ Yes $)$ & & & & $\begin{array}{l}1.50(0.206) \\
(0.80,2.81)\end{array}$ \\
\hline & Wald (p) & $7.59(0.108)$ & $37.66(<0.001)$ & $41.83(<0.001)$ & $44.87(<0.001)$ \\
\hline & Nagelkerke's R ${ }^{2}$ & 0.039 & 0.213 & 0.245 & 0.272 \\
\hline
\end{tabular}


Table 4. Factors affecting usual activity dimension of health-related quality of life in patients with multimorbidity according to Andersen's model (South Korea, 2014-2016).

\begin{tabular}{|c|c|c|c|c|c|}
\hline \multirow{2}{*}{\multicolumn{2}{|c|}{ Variable }} & Model 1 & Model 2 & Model 3 & Model 4 \\
\hline & & $\begin{array}{l}\text { OR }(p) \\
(95 \% \mathrm{CI})\end{array}$ & $\begin{array}{c}\text { OR }(p) \\
(95 \% \text { CI })\end{array}$ & $\begin{array}{l}\text { OR }(p) \\
(95 \% \text { CI })\end{array}$ & $\begin{array}{c}\text { OR }(p) \\
(95 \% \text { CI })\end{array}$ \\
\hline \multirow{4}{*}{$\begin{array}{l}\text { Predisposing } \\
\text { factors }\end{array}$} & Gender (ref = Male) & $\begin{array}{l}1.01(0.973) \\
(0.63,1.62)\end{array}$ & $\begin{array}{l}1.15(0.590) \\
(0.69,1.93)\end{array}$ & $\begin{array}{l}1.03(0.921) \\
(0.60,1.76)\end{array}$ & $\begin{array}{l}1.29(0.425) \\
(0.69,2.39)\end{array}$ \\
\hline & Age (ref = Under 65) & $\begin{array}{l}1.47(0.144) \\
(0.88,2.45)\end{array}$ & $\begin{array}{l}1.09(0.772) \\
(0.60,1.99)\end{array}$ & $\begin{array}{l}1.26(0.474) \\
(0.67,2.34)\end{array}$ & $\begin{array}{l}1.40(0.313) \\
(0.73,2.71)\end{array}$ \\
\hline & $\begin{array}{c}\text { Education level } \\
(\text { ref = Below high school } \\
\text { graduate })\end{array}$ & $\begin{array}{l}0.51(0.074) \\
(0.24,1.07)\end{array}$ & $\begin{array}{l}0.67(0.328) \\
(0.29,1.51)\end{array}$ & $\begin{array}{l}0.80(0.609) \\
(0.34,1.88)\end{array}$ & $\begin{array}{l}0.77(0.581) \\
(0.31,1.92)\end{array}$ \\
\hline & Marital status (ref = With spouse) & $\begin{array}{l}1.46(0.136) \\
(0.89,2.41)\end{array}$ & $\begin{array}{l}1.43(0.346) \\
(0.68,2.97)\end{array}$ & $\begin{array}{l}1.49(0.306) \\
(0.70,3.19)\end{array}$ & $\begin{array}{l}1.31(0.513) \\
(0.58,2.94)\end{array}$ \\
\hline \multirow{7}{*}{ Enabling factors } & Basic living security (ref = No) & & $\begin{array}{l}0.37(0.065) \\
(0.13,1.07)\end{array}$ & $\begin{array}{l}0.31(0.038) \\
(0.10,0.94)\end{array}$ & $\begin{array}{l}0.30(0.037) \\
(0.10,0.93)\end{array}$ \\
\hline & $\begin{array}{l}\text { Health insurance (ref = National } \\
\text { health insurance) }\end{array}$ & & $\begin{array}{l}3.13(0.050) \\
(1.00,9.82)\end{array}$ & $\begin{array}{r}3.75(0.033) \\
(1.12,12.60)\end{array}$ & $\begin{array}{l}3.15(0.063) \\
(0.94,10.56)\end{array}$ \\
\hline & Private insurance $(\mathrm{ref}=\mathrm{Yes})$ & & $\begin{array}{l}1.81(0.040) \\
(1.03,3.19)\end{array}$ & $\begin{array}{l}1.77(0.056) \\
(0.99,3.19)\end{array}$ & $\begin{array}{l}1.83(0.054) \\
(0.99,3.37)\end{array}$ \\
\hline & \multirow{2}{*}{$\begin{array}{l}\text { Household income (monthly } \\
\text { average) }\end{array}$} & & $1.00(0.525)$ & $1.00(0.628)$ & $1.00(0.943)$ \\
\hline & & & $(1.00,1.00)$ & $(1.00,1.00)$ & $(1.00,1.00)$ \\
\hline & Living with family (ref = Yes) & & $\begin{array}{l}0.84(0.688) \\
(0.37,1.94)\end{array}$ & $\begin{array}{l}0.89(0.798) \\
(0.38,2.11)\end{array}$ & $\begin{array}{l}1.05(0.921) \\
(0.43,2.54)\end{array}$ \\
\hline & Limited activity (ref = No) & & $\begin{array}{l}5.05(<0.001) \\
(2.87,8.87)\end{array}$ & $\begin{array}{c}3.69(<0.001) \\
(2.03,6.69)\end{array}$ & $\begin{array}{l}4.22(<0.001) \\
(2.26,7.89)\end{array}$ \\
\hline \multirow{3}{*}{ Need factors } & Number of chronic diseases & & & \multirow{3}{*}{$\begin{array}{c}1.49(0.186) \\
(0.83,2.67) \\
3.05(<0.001) \\
(1.75,5.34) \\
1.13(0.763) \\
(0.50,2.57)\end{array}$} & $\begin{array}{l}1.55(0.159) \\
(0.84,2.86)\end{array}$ \\
\hline & $\begin{array}{l}\text { Subjective health status (ref }= \\
\text { Good) }\end{array}$ & & & & $\begin{array}{l}2.81(<0.001) \\
(1.58,5.00)\end{array}$ \\
\hline & Unmet medical needs $(\mathrm{ref}=\mathrm{No})$ & & & & $\begin{array}{l}1.26(0.588) \\
(0.54,2.94)\end{array}$ \\
\hline \multirow{4}{*}{ Health behaviors } & Smoking $(\mathrm{ref}=\mathrm{No})$ & & & & $\begin{array}{l}2.56(0.013) \\
(1.22,5.38)\end{array}$ \\
\hline & Drinking $(\mathrm{ref}=\mathrm{No})$ & & & & $\begin{array}{l}0.91(0.757) \\
(0.50,1.65)\end{array}$ \\
\hline & Physical activity (ref = Yes) & & & & $\begin{array}{l}0.85(0.700) \\
(0.36,1.98)\end{array}$ \\
\hline & Health screenings (ref = Yes) & & & & $\begin{array}{l}1.69(0.061) \\
(0.98,2.93)\end{array}$ \\
\hline & Wald (p) & $8.83(0.066)$ & $46.88(<0.001)$ & $57.93(<0.001)$ & $63.39(<0.001)$ \\
\hline & Nagelkerke's R ${ }^{2}$ & 0.038 & 0.225 & 0.291 & 0.331 \\
\hline
\end{tabular}


Table 5. Factors affecting pain/discomfort dimension of health-related quality of life in patients with multimorbidity according to Andersen's model (South Korea, 2014-2016).

\begin{tabular}{|c|c|c|c|c|c|}
\hline \multirow{2}{*}{\multicolumn{2}{|c|}{ Variable }} & Model 1 & Model 2 & Model 3 & Model 4 \\
\hline & & $\begin{array}{c}\text { OR }(p) \\
(95 \% \text { CI })\end{array}$ & $\begin{array}{c}\text { OR }(p) \\
(95 \% \text { CI })\end{array}$ & $\begin{array}{c}\text { OR }(p) \\
(95 \% \text { CI })\end{array}$ & $\begin{array}{c}\text { OR }(p) \\
(95 \% \text { CI) }\end{array}$ \\
\hline \multirow{4}{*}{$\begin{array}{l}\text { Predisposing } \\
\text { factors }\end{array}$} & Gender (ref = Male) & $\begin{array}{l}1.76(0.018) \\
(1.10,2.82)\end{array}$ & $\begin{array}{l}2.14(0.003) \\
(1.28,3.56)\end{array}$ & $\begin{array}{l}2.04(0.008) \\
(1.21,3.43)\end{array}$ & $\begin{array}{l}2.14(0.012) \\
(1.19,3.87)\end{array}$ \\
\hline & Age (ref = Under 65) & $\begin{array}{l}1.25(0.398) \\
(0.75,2.07)\end{array}$ & $\begin{array}{l}1.37(0.296) \\
(0.76,2.48)\end{array}$ & $\begin{array}{l}1.59(0.138) \\
(0.86,2.93)\end{array}$ & $\begin{array}{l}1.68(0.108) \\
(0.89,3.18)\end{array}$ \\
\hline & $\begin{array}{c}\text { Education level } \\
(\text { ref = Below high school } \\
\text { graduate })\end{array}$ & $\begin{array}{l}0.56(0.120) \\
(0.27,1.16)\end{array}$ & $\begin{array}{l}0.54(0.142) \\
(0.24,1.23)\end{array}$ & $\begin{array}{l}0.64(0.284) \\
(0.28,1.46)\end{array}$ & $\begin{array}{l}0.57(0.212) \\
(0.24,1.38)\end{array}$ \\
\hline & Marital status (ref = With spouse) & $\begin{array}{l}1.43(0.158) \\
(0.87,2.37)\end{array}$ & $\begin{array}{l}1.14(0.716) \\
(0.56,2.33)\end{array}$ & $\begin{array}{l}1.23(0.579) \\
(0.59,2.56)\end{array}$ & $\begin{array}{l}1.20(0.636) \\
(0.56,2.56)\end{array}$ \\
\hline \multirow{6}{*}{ Enabling factors } & Basic living security (ref = No) & & $\begin{array}{l}0.51(0.185) \\
(0.18,1.39)\end{array}$ & $\begin{array}{l}0.42(0.109) \\
(0.15,1.21)\end{array}$ & $\begin{array}{l}0.41(0.099) \\
(0.14,1.18)\end{array}$ \\
\hline & $\begin{array}{c}\text { Health insurance }(\text { ref }=\text { National } \\
\text { health insurance) }\end{array}$ & & $\begin{array}{r}5.14(0.005) \\
(1.63,16.25)\end{array}$ & $\begin{array}{l}5.87(0.004) \\
(1.76,19.58)\end{array}$ & $\begin{array}{r}5.31(0.007) \\
(1.58,17.88)\end{array}$ \\
\hline & Private insurance $($ ref $=$ Yes) & & $\begin{array}{l}0.90(0.709) \\
(0.51,1.58)\end{array}$ & $\begin{array}{l}0.86(0.593) \\
(0.48,1.52)\end{array}$ & $\begin{array}{l}0.83(0.536) \\
(0.46,1.49)\end{array}$ \\
\hline & $\begin{array}{c}\text { Household income (monthly } \\
\text { average) }\end{array}$ & & $\begin{array}{l}1.00(0.150) \\
(1.00,1.00)\end{array}$ & $\begin{array}{l}1.00(0.102) \\
(1.00,1.00)\end{array}$ & $\begin{array}{l}1.00(0.074) \\
(1.00,1.00)\end{array}$ \\
\hline & Living with family (ref = Yes) & & $\begin{array}{l}1.40(0.419) \\
(0.62,3.15)\end{array}$ & $\begin{array}{l}1.46(0.373) \\
(0.34,3.32)\end{array}$ & $\begin{array}{l}1.61(0.267) \\
(0.69,3.75)\end{array}$ \\
\hline & Limited activity $(\mathrm{ref}=\mathrm{No})$ & & $\begin{array}{c}4.44(<0.001) \\
(2.50,7.89)\end{array}$ & $\begin{array}{c}3.29(<0.001) \\
(1.80,6.02)\end{array}$ & $\begin{array}{c}3.64(<0.001) \\
(1.94,6.81)\end{array}$ \\
\hline \multirow{3}{*}{ Need factors } & Number of chronic diseases & & & $\begin{array}{l}1.27(0.423) \\
(0.71,2.28)\end{array}$ & $\begin{array}{l}1.29(0.402) \\
(0.71,2.36)\end{array}$ \\
\hline & $\begin{array}{l}\text { Subjective health status }(\mathrm{ref}= \\
\text { Good) }\end{array}$ & & & $\begin{array}{l}2.17(0.005) \\
(1.26,3.72)\end{array}$ & $\begin{array}{l}2.05(0.011) \\
(1.18,3.58)\end{array}$ \\
\hline & Unmet medical needs $(\mathrm{ref}=\mathrm{No})$ & & & $\begin{array}{l}1.96(0.106) \\
(0.87,4.44)\end{array}$ & $\begin{array}{l}2.08(0.086) \\
(0.90,4.79) \\
\end{array}$ \\
\hline \multirow{4}{*}{ Health behaviors } & Smoking $(\mathrm{ref}=\mathrm{No})$ & & & & $\begin{array}{l}1.48(0.290) \\
(0.72,3.05)\end{array}$ \\
\hline & Drinking $(\mathrm{ref}=\mathrm{No})$ & & & & $\begin{array}{l}0.81(0.464) \\
(0.46,1.42)\end{array}$ \\
\hline & Physical activity (ref = Yes) & & & & $\begin{array}{l}0.64(0.279) \\
(0.28,1.44)\end{array}$ \\
\hline & Health screenings $($ ref = Yes) & & & & $\begin{array}{l}1.13(0.661) \\
(0.66,1.92)\end{array}$ \\
\hline & Wald (p) & $15.36(0.004)$ & $45.96(<0.001)$ & $53.00(<0.001)$ & $55.16(<0.001)$ \\
\hline & Nagelkerke's $\mathrm{R}^{2}$ & 0.066 & 0.227 & 0.272 & 0.292 \\
\hline
\end{tabular}


Table 6. Factors affecting anxiety/depression dimension of health-related quality of life in patients with multimorbidity according to Andersen's model (South Korea, 2014-2016).

\begin{tabular}{|c|c|c|c|c|c|}
\hline \multirow{2}{*}{\multicolumn{2}{|c|}{ Variable }} & Model 1 & Model 2 & Model 3 & Model 4 \\
\hline & & $\begin{array}{c}\text { OR }(p) \\
(95 \% \text { CI })\end{array}$ & $\begin{array}{c}\text { OR }(p) \\
(95 \% \text { CI })\end{array}$ & $\begin{array}{c}\text { OR }(p) \\
(95 \% \text { CI })\end{array}$ & $\begin{array}{c}\text { OR }(p) \\
(95 \% \text { CI })\end{array}$ \\
\hline \multirow{4}{*}{$\begin{array}{l}\text { Predisposing } \\
\text { factors }\end{array}$} & Gender (ref = Male) & $\begin{array}{l}1.18(0.536) \\
(0.70,2.00)\end{array}$ & $\begin{array}{l}1.20(0.514) \\
(0.69,2.08)\end{array}$ & $\begin{array}{l}1.10(0.754) \\
(0.62,1.94)\end{array}$ & $\begin{array}{l}1.27(0.469) \\
(0.66,2.43)\end{array}$ \\
\hline & Age (ref = Under 65) & $\begin{array}{l}0.60(0.062) \\
(0.35,1.03)\end{array}$ & $\begin{array}{l}0.54(0.048) \\
(0.29,1.00)\end{array}$ & $\begin{array}{l}0.62(0.141) \\
(0.33,1.17)\end{array}$ & $\begin{array}{l}0.67(0.229) \\
(0.35,1.29)\end{array}$ \\
\hline & $\begin{array}{c}\text { Education level } \\
(\text { ref = Below high school } \\
\text { graduate })\end{array}$ & $\begin{array}{l}0.71(0.413) \\
(0.31,1.63)\end{array}$ & $\begin{array}{l}0.86(0.742) \\
(0.36,2.08)\end{array}$ & $\begin{array}{l}1.03(0.957) \\
(0.41,2.53)\end{array}$ & $\begin{array}{l}0.94(0.892) \\
(0.37,2.41)\end{array}$ \\
\hline & Marital status (ref $=$ With spouse) & $\begin{array}{l}1.26(0.417) \\
(0.73,2.18)\end{array}$ & $\begin{array}{l}1.09(0.833) \\
(0.50,2.37)\end{array}$ & $\begin{array}{l}1.17(0.702) \\
(0.52,2.63)\end{array}$ & $\begin{array}{l}1.20(0.664) \\
(0.52,2.78)\end{array}$ \\
\hline \multirow{6}{*}{ Enabling factors } & Basic living security (ref = No) & & $\begin{array}{l}0.63(0.386) \\
(0.22,1.80)\end{array}$ & $\begin{array}{l}0.51(0.239) \\
(0.17,1.56)\end{array}$ & $\begin{array}{l}0.53(0.269) \\
(0.17,1.63)\end{array}$ \\
\hline & $\begin{array}{l}\text { Health insurance }(\mathrm{ref}=\text { national } \\
\text { health insurance) }\end{array}$ & & $\begin{array}{l}2.18(0.164) \\
(0.73,6.52)\end{array}$ & $\begin{array}{l}2.18(0.184) \\
(0.69,6.90)\end{array}$ & $\begin{array}{l}2.16(0.202) \\
(0.66,7.03)\end{array}$ \\
\hline & Private insurance $($ ref = Yes) & & $\begin{array}{l}0.85(0.607) \\
(0.47,1.56)\end{array}$ & $\begin{array}{l}0.82(0.524) \\
(0.44,1.51)\end{array}$ & $\begin{array}{l}0.83(0.549) \\
(0.45,1.54)\end{array}$ \\
\hline & $\begin{array}{l}\text { Household income (monthly } \\
\text { average) }\end{array}$ & & $\begin{array}{l}1.00(0.509) \\
(1.00,1.00)\end{array}$ & $\begin{array}{l}1.00(0.778) \\
(1.00,1.00)\end{array}$ & $\begin{array}{l}1.00(0.804) \\
(1.00,1.00)\end{array}$ \\
\hline & Living with family (ref = Yes) & & $\begin{array}{l}0.92(0.860) \\
(0.38,2.23)\end{array}$ & $\begin{array}{l}1.04(0.936) \\
(0.42,2.55)\end{array}$ & $\begin{array}{l}1.01(0.985) \\
(0.40,2.53)\end{array}$ \\
\hline & Limited activity $(\mathrm{ref}=\mathrm{No})$ & & $\begin{array}{c}2.74(<0.001) \\
(1.57,4.79)\end{array}$ & $\begin{array}{l}1.91(0.037) \\
(1.04,3.50)\end{array}$ & $\begin{array}{l}2.04(0.024) \\
(1.10,3.80)\end{array}$ \\
\hline \multirow{3}{*}{ Need factors } & Number of chronic diseases & & & $\begin{array}{l}1.07(0.826) \\
(0.58,1.97)\end{array}$ & $\begin{array}{l}1.05(0.875) \\
(0.57,1.95)\end{array}$ \\
\hline & $\begin{array}{l}\text { Subjective health status }(\mathrm{ref}= \\
\text { Good) }\end{array}$ & & & $\begin{array}{l}2.33(0.009) \\
(1.23,4.39)\end{array}$ & $\begin{array}{l}2.26(0.013) \\
(1.19,4.32)\end{array}$ \\
\hline & Unmet medical needs $($ ref $=\mathrm{No})$ & & & $\begin{array}{l}2.91(0.006) \\
(1.35,6.29)\end{array}$ & $\begin{array}{l}2.95(0.007) \\
(1.35,6.47)\end{array}$ \\
\hline \multirow{4}{*}{ Health behaviors } & Smoking $(\mathrm{ref}=\mathrm{No})$ & & & & $\begin{array}{l}1.54(0.258) \\
(0.73,3.23)\end{array}$ \\
\hline & Drinking $(\mathrm{ref}=\mathrm{No})$ & & & & $\begin{array}{l}1.06(0.863) \\
(0.57,1.97)\end{array}$ \\
\hline & Physical activity (ref = Yes) & & & & $\begin{array}{l}0.72(0.434) \\
(0.32,1.64)\end{array}$ \\
\hline & Health screenings $(\mathrm{ref}=\mathrm{Yes})$ & & & & $\begin{array}{l}0.95(0.867) \\
(0.54,1.68)\end{array}$ \\
\hline & Wald (p) & $5.49(0.241)$ & $19.96(0.030)$ & $31.82(0.003)$ & $33.48(0.010)$ \\
\hline & Nagelkerke's R ${ }^{2}$ & 0.025 & 0.095 & 0.165 & 0.178 \\
\hline
\end{tabular}

OR: odds ratio; CI: confidence interval.

\section{Discussion}

The public health sector in Korea is currently facing rapid population aging with the consequent increase in prevalent multimorbidity and related medical expenses. Despite the long average life expectancy in Korea, chronic diseases contribute to a relatively low healthy life expectancy; thus, increasing the QoL of patients with multimorbidity may be an important policy issue. Previous studies on multimorbidity concentrated on overall HRQoL (EQ-5D index) [27], whereas recent studies have placed more importance on examining each dimension [28,29]. As QoL is a complex concept encompassing all elements for a satisfactory life [30], it is necessary to develop specific strategies to improve each dimension of QoL by inspecting the factors that influence it. In our study, Model 4, which included all of the predisposing, enabling, need factors, and health behaviors, had the greatest influence 
on all five dimensions. This indicates that all four factor types must be considered when examining HRQoL.

Regarding the specific HRQoL dimensions, in Models 1-4, respondents $\geq 65$ years, without private insurance, with limited activity, with poor subjective health status, and/or with unmet medical needs were more likely to have problems with mobility. Our results support those from previous studies suggesting that physical function declines with age, thereby reducing QoL in elderly persons [31]. Having unmet medical needs, which refers to cases where treatment/examination is needed but not received, was attributable to time limitations as well as poor access to healthcare institutions.

As for self-care, no factors had statistical significance in Model 1, whereas in Models $2-4$, respondents without private insurance $(63.5 \%)$ and / or with limited activity were more likely to have problems. This implies that most of these patients did not have a secure financial status, which is supported by the fact that $49.1 \%$ of the respondents were at the bottom end of the income distribution. Those with limited activity were more likely to have problems with self-care, which can be related to poor financial conditions, since limited activity is related to low income [32].

Regarding usual activity, no factors had statistical significance in Model 1, whereas in Models 2-4, respondents who had never received basic living security, who received Medicaid, and/or without private insurance were more likely to have problems, which suggests that poor financial status, indicated by Medicaid and lack of private insurance, leads to decreased ability to perform usual activities. Although a social guarantee system such as basic living security is limited, it can still improve basic QoL. This implies that a policy that includes individuals who are not covered by such social systems or private insurance needs to be implemented. In Model 4, respondents who had never received basic living security, with limited activity, who had poor subjective health status, and/or who smoked were more likely to have problems with usual activities. As an individual habitual health-related behavior, smoking affected daily activities (work, studying, housework, and leisure) across all five dimensions of the HRQoL. Smoking must be strictly controlled, as it is one of the major causes of chronic disease, ultimately leading to multimorbidity. A special smoking cessation program for patients with multimorbidity together with an efficient stress management program may be helpful for patients with multimorbidity who have difficulties with smoking cessation. Moreover, subjective health status involves a comprehensive awareness of one's health and the social environment surrounding it; therefore, establishing social systems that improve these subjective health conditions is necessary to increase daily activity in patients with multimorbidity.

Regarding pain/discomfort, gender was a significant factor in Model 1, and gender, type of insurance, and limited activity were significant in Model 2. In Models 3 and 4, respondents who were female, who received Medicaid, with limited activity, and/or with poor subjective health status were more likely to experience pain/discomfort. This is in line with the results of previous studies reporting that females are more likely to experience pain related to musculoskeletal, circulatory, respiratory, and digestive systems [33]. A management system for patients with multimorbidity must be established considering differences in pain/discomfort, and gender is an important factor to take into account, since studies conducted in other countries using the EQ-5D also showed that females were more likely to experience difficulties in this domain.

According to studies on multimorbidity and QoL, multimorbidity has an impact on the physical dimensions of QoL, but little is known about the effect on social and emotional dimensions of QoL [34]; thus, assessing the anxiety/depression domain is especially important for patients with multimorbidity. No factors were significant in Model 1, whereas in Model 2, age and limited activity were significant. In Models 3 and 4, respondents with limited activity, with poor subjective health status, and/or with unmet medical needs were more likely to experience anxiety/depression. According to a study on patients with chronic diseases by the Korea Health Panel [32], vulnerable social groups (older persons, those with low income, persons with disabilities, and those with a low 
educational level) had a higher level of limited activity and unmet medical needs; moreover, patients with chronic diseases who had limited activity were more likely to have unmet medical needs than those without limited activity. The percentage of respondents with multimorbidity and unmet medical needs in the present study was $12.5 \%$, which is lower than $23.7 \%$ among young adults and $21.7 \%$ among older adults reported by the Korea Health Panel [27]. Additionally, our participants had an average of 3.2 chronic diseases, and no participant had more than 6 chronic diseases, whereas the study conducted by Korea Health Panel reported a mean of 5.75 diseases, and $31.2 \%$ of the study participants had at least 7 chronic diseases. Considering such results and the influence of limited activity and unmet medical needs on anxiety/depression in patients with multimorbidity, adequate healthcare services and activity support programs must be provided for these patients. Moreover, measures to improve individuals' subjective health status must be considered.

Those with limited activity were more likely to have problems with mobility, selfcare, usual activities, pain/discomfort, and anxiety/depression. Hence, interventions to assist or improve the limitations in activity of patients with multimorbidity must be implemented with the highest priority. Specific strategies, such as visiting healthcare or medical services, leisure activity assistance programs, or transportation assistance programs for multimorbidity patients with limited activity must be established. Access to healthcare must be improved to prevent unmet medical needs of those with limited activity.

For multimorbidity patients with multiple chronic diseases, some suggest that another level of treatment deviating from existing treatment methods must be provided, as they regard multimorbidity as a systematic disease where chronic diseases affect each other, rather than a combination of independent chronic diseases [35]. The results of this study are meaningful in this respect, as we targeted patients with multimorbidity according to the standards of the WHO; accordingly, in a study targeting the general population, patients with at least three chronic diseases are considered to have multimorbidity; however, we did not include certain chronic diseases such as chronic obstructive pulmonary disease. Studies drawn from a broader population of patients should thus be conducted in the future. Although we used data from a reliable and representative large-scale national dataset, not all the dimension for Andersen's model were met. Furthermore, the design of our study was cross-sectional, which limits interpretations regarding causality.

\section{Conclusions and Recommendation}

Predisposing, enabling, and need factors along with health behaviors need to be examined when investigating the HRQoL of patients with multimorbidity, with a focus on the specific HRQoL dimensions in order to further provide personalized care. Individuals with limited activity were more likely to experience problems with mobility, self-care, usual activities, pain/discomfort, and anxiety/depression; hence, interventions to address the limited activity of these patients should be a top priority.

In Korea, the frequency of using healthcare services may increase through self-referral or cross-referral when treating multimorbidity due to the limitations of the primary care system [8]. Patients may still experience unmet medical needs despite excessive healthcare service use. Therefore, multimorbidity needs to be treated in primary care. In one pilot project conducted in Korea, the results showed that a multimorbidity support center model based on primary care could efficiently manage multimorbidity patients and reduce the use of unnecessary medical services by controlling the selection of appropriate targets, multidisciplinary management of healthcare fields, establishing a personalized care plan for each patient, and integrating treatment and patient education [35].

Author Contributions: E.L. was involved in the study design, statistical analysis, and description of study methods and results. G.M.K. was involved in the literature review and writing the manuscript. S.C. was involved in the study design, literature review, and writing the manuscript. E.L., G.M.K. and S.C. also made substantial contributions to the draft and critical revision of the manuscript. All authors listed meet the authorship criteria according to the latest guidelines of the International 
Committee of Medical Journal Editors. All authors have read and agreed to the published version of the manuscript.

Funding: This work was supported by the Sun Moon University Research Grant of 2019.

Institutional Review Board Statement: The study was approved by the Sun Moon University's Institutional Review Board (No. SM-201904-010-1). Ethical issues regarding plagiarism, informed consent, misconduct, data fabrication and/or falsification, double publication and/or submission, and redundancy have been completely observed by the authors.

Informed Consent Statement: Not applicable.

Data Availability Statement: Data were obtained from KCDC and are available from https:/ / knhanes.cdc.go.kr/knhanes/sub03/sub03_02_05.do (accessed on 5 September 2018).

Acknowledgments: The authors thank the Korea Centers for Disease Control and Prevention for providing the study data.

Conflicts of Interest: The authors declare that they have no conflict of interest.

\section{References}

1. Organisation for Economic Co-Operation and Development. OECD Health Care Quality Review: Korea. OECD 2012. Available online: https:/ / www.oecd.org/els/health-systems/49818570.pdf (accessed on 24 June 2019).

2. OECD. Health Reform: Meeting the Challenge of Ageing and Multiple Morbidities; OECD Publishing: Paris, France, 2011.

3. Akker, M.V.D.; Buntinx, F.; Metsemakers, J.F.; Roos, S.; Knottnerus, J. Multimorbidity in General Practice: Prevalence, Incidence, and Determinants of Co-Occurring Chronic and Recurrent Diseases. J. Clin. Epidemiol. 1998, 51, 367-375. [CrossRef]

4. $\quad$ Bayliss, E.A.; Steiner, J.F.; Fernald, D.H.; Crane, L.A.; Main, D.S. Descriptions of Barriers to Self-Care by Persons with Comorbid Chronic Diseases. Ann. Fam. Med. 2003, 1, 15-21. [CrossRef] [PubMed]

5. Bussche, H.V.D.; Koller, D.; Kolonko, T.; Hansen, H.; Wegscheider, K.; Glaeske, G.; Von Leitner, E.-C.; Schäfer, I.; Schön, G. Which chronic diseases and disease combinations are specific to multimorbidity in the elderly? Results of a claims data based cross-sectional study in Germany. BMC Public Health 2011, 11, 101. [CrossRef]

6. Barnett, K.; Mercer, S.W.; Norbury, M.; Watt, G.; Wyke, S.; Guthrie, B. Epidemiology of multimorbidity and implications for health care, research, and medical education: A cross-sectional study. Lancet 2012, 380, 37-43. [CrossRef]

7. Jung, Y.H.; Go, S.J.; Kim, E.J. A Study on the Effective Chronic Disease Management; Korea Institute for Health and Social Affairs: Sicheong-daero, Korea, 2013; Available online: https:/ / data.kihasa.re.kr/researchdb/subject/view? seq=30 (accessed on $24 \mathrm{June}$ 2019).

8. Kim, C.-H.; Hwang, I.; Yoo, W.-S. The Common Patterns of Multimorbidity and Its Impact on Healthcare Cost in Korea. Heal. Policy Manag. 2014, 24, 219-227. [CrossRef]

9. Lehnert, T.; Heider, D.; Leicht, H.; Heinrich, S.; Corrieri, S.; Luppa, M.; Riedel-Heller, S.; König, H.-H. Review: Health Care Utilization and Costs of Elderly Persons with Multiple Chronic Conditions. Med Care Res. Rev. 2011, 68, 387-420. [CrossRef]

10. Kim, C.; Lee, M.; Yoo, W. Common combinations of chronic diseases in multimorbidity and their effect on healthcare cost in Korea. Korean J. Health Econ. Policy 2014, 20, 81-102.

11. Steiner, C.A.; Friedman, B. Hospital Utilization, Costs, and Mortality for Adults with Multiple Chronic Conditions, Nationwide Inpatient Sample, 2009. Prev. Chronic Dis. 2013, 10, E62. [CrossRef]

12. DeLateur, B.J. Quality of life: A patient-centered outcome. Arch. Phys. Med. Rehabil. 1997, 78, 237-239. [CrossRef]

13. Nam, H.S.; Son, M.H.; Kweon, S.S.; Kim, S.Y.; Park, H.C.; Lee, C.W.; Choi, J.S. Chronic disease and health-related quality of life in an urban elderlies. J. Korean Geriatr. Soc. 2000, 4, 172-190.

14. Choi, Y.J. A Study on Factors That Affect Quality of Life of the Elderly by Age. Ph.D. Thesis, Hanyoung The-Ological University, Seoul, Korea, 2008.

15. Kil, S.R.; Lee, S.-I.; Yun, S.-C.; An, H.-M.; Jo, M.-W. The Decline of Health-Related Quality of Life Associated with Some Diseases in Korean Adults. J. Prev. Med. Public Health 2008, 41, 434. [CrossRef]

16. Ryu, Y.M. A Study on the Factors Influencing the Quality of Life of the Old: A Case of Yongin City. Master's Thesis, Ajou University, Suwon, Korea, 2009.

17. Yoon, S.D.; Han, G.H. Productive activities and psychological well-being of the rural elderly in Korea. J. Korea Gerontol. Soc. 2004, 24, 57-77.

18. Andersen, R.M. National Health Surveys and the Behavioral Model of Health Services Use. Med Care 2008, 46, 647-653. [CrossRef] [PubMed]

19. Kim, Y.S.; Park, B.H.; Lee, H.Y. A study on predicting health literacy of Korean elderly using Andersen's health behavior model. Korean J. Gerontol. Soc. Welf. 2014, 65, 35-57.

20. Kim, G.M.; Hong, M.S.; Noh, W. Factors affecting the health-related quality of life in community-dwelling elderly people. Public Health Nurs. 2018, 35, 482-489. [CrossRef] 
21. Yom, Y.-H.; Han, J.-H. Factors Associated with Oral Health Related-quality of Life in Elderly Persons: Applying Andersen's Model. J. Korean Acad. Fundam. Nurs. 2014, 21, 18-28. [CrossRef]

22. Andersen, R.M.; Davidson, P.L.; Baumeister, S.E. Improving access to care. In Changing the US Health Care System: Key Issues in Health Services Policy and Management, 4th ed.; Kominski, G., Ed.; Jossey-Bass: San Francisco, CA, USA, 2013 ; pp. 33-69.

23. Andersen, R.M. Revisiting the Behavioral Model and Access to Medical Care: Does it Matter? J. Heal. Soc. Behav. 1995, 36, 1-10. [CrossRef]

24. Park, J.D. A Study on Factors Influencing Solitary Home-Staying Elders' Intention to Participate in Leisure Programs-An Application of Anderson Model Focused on Health Promotion Activity Program. Master's Thesis, Sogang University, Seoul, Korea, 2010. Unpublished.

25. Kim, S.I. A Study on the Causes Which Have Impact on the Recognition Rehabilitation Service Use Intention of the Elderly with Dementia. Master's Thesis, Catholic Kwan-Dong University, Gangneug, Korea, 2015.

26. Korea Centers for Disease Control and Prevention. The Seventh Korea National Health and Nutrition Examination Survey 2016; KCDC: Seoul, Korea, 2017.

27. Joe, S.; Lee, I.; Park, B. Factors Influencing Health-related Quality of Life of Young Adults and Elderly with Multimorbiditiy: A Secondary Analysis of the 2013 Korea Health Panel Data. J. Korean Acad. Community Health Nurs. 2016, 27, 358-369. [CrossRef]

28. Alcañiz, M.; Solé-Auró, A. Feeling good in old age: Factors explaining health-related quality of life. Heal. Qual. Life Outcomes 2018, 16, 48. [CrossRef] [PubMed]

29. Ophuis, R.H.; Janssen, M.F.; Bonsel, G.J.; Panneman, M.J.; Polinder, S.; Haagsma, J.A. Health-related quality of life in injury patients: The added value of extending the EQ-5D-3L with a cognitive dimension. Qual. Life Res. 2019, 28, 1941-1949. [CrossRef] [PubMed]

30. Nam, S.K.; Shim, O.S. A study on the influence factors on quality of life of elderly with chronic disease. J. Welf. Aged. 2011, 53, 196-216.

31. Kim, J.I. Levels of health-related quality of life (EQ-5D) and its related factors among vulnerable elders receiving home visiting health care services in some rural areas. J. Korean Acad. Community Health Nurs. 2013, 24, 99-109. [CrossRef]

32. Jung, Y.H. A report on the Korea health panel survey: Restrictions on activities and unmet medical needs. Issue Focus 2012, 120, $1-8$.

33. Ruau, D.; Liu, L.Y.; Clark, J.D.; Angst, M.S.; Butte, A.J. Sex Differences in Reported Pain Across 11,000 Patients Captured in Electronic Medical Records. J. Pain 2012, 13, 228-234. [CrossRef] [PubMed]

34. Fortin, M.; Lapointe, L.; Hudon, C.; Vanasse, A.; Ntetu, A.L.; Maltais, D. Multimorbidity and quality of life in primary care: A systematic review. Health Qual. Life Outcomes 2004, 2, 51. [CrossRef] [PubMed]

35. Lee, S.H.; Park, S.B.; Cho, K.H.; Choi, Y.E.; Choi, J.H.; Han, K.T.; Choo, J.E.; Kim, Y.S. A Study on the Effect Analysis and Management of the Pilot Project for the Multimorbidity; National Health Insurance Service Ilsan Hospital: Goyang-si, Korea, 2019; Available online: http:/ / www.alio.go.kr/popSusiViewB1040.do (accessed on 24 June 2019). 